\title{
A 7-Amino-Acid Motif of Rep Protein Essential for Virulence Is Critical for Triggering Host Defense Against Sri Lankan Cassava Mosaic Virus
}

\author{
Duan Wang, ${ }^{1,2}$ Xuan Zhang, ${ }^{1}$ Xiangmei Yao, ${ }^{1}$ Peng Zhang, ${ }^{3}$ Rongxiang Fang, ${ }^{1,2}$ and Jian Ye ${ }^{1,2,+}$ \\ ${ }^{1}$ State Key Laboratory of Plant Genomics, Institute of Microbiology, Chinese Academy of Sciences, Beijing 100101, China \\ ${ }^{2}$ CAS Center for Excellence in Biotic Interactions, University of the Chinese Academy of Sciences, Beijing 100049, China \\ ${ }^{3}$ Institute of Plant Physiology and Ecology, Shanghai Institutes for Biological Sciences, Chinese Academy of Sciences
}

Accepted 4 September 2019.

\begin{abstract}
Geminiviruses cause severe damage to agriculture worldwide. The replication (Rep) protein is the indispensable viral protein for viral replication. Although various functional domains of Rep protein in Geminivirus spp. have been characterized, the most carboxyl terminus of Rep protein was not available. We have reported the first cassava-infecting geminivirus, Sri Lankan cassava mosaic virus (SLCMV-HN7 strain), in China. In this study, we reported the second Chinese SLCMV strain, SLCMV-Col, and conducted comparative genomic analysis between these two SLCMV strains. The virulence of SLCMVCol is much stronger than SLCMV-HN7, indicated by the higher virus titer, more severe symptoms, and more extent host defense. We functionally characterized that Rep protein, a 7-amino-acid motif at the most carboxyl terminus, is essential for Rep protein accumulation and virulence of SLCMV. We also provided evidence suggesting that the motif could also enhance triggering of salicylic acid (SA) defense against SLCMV infection in Nicotiana benthamiana. The significance of the balance between virulence and host $\mathrm{SA}$ defense responses in expanding invasions of SLCMV is also discussed.
\end{abstract}

Keywords: virus-plant interactions

Geminiviridae is the largest family of the plant-infecting viruses. It has been classified into nine genera and two unassigned species based on their genome, insect vector, and host ranges (Varsani et al. 2017; Zerbini et al. 2017). As a class of singlestrand DNA virus, geminivirus has a limited coding capability and most open reading frames (ORFs) are overlapping. Among six to eight ORFs encoding for the Geminivirus genome, the replication (Rep) protein coded by the $\mathrm{AC} 1 / \mathrm{C} 1 \mathrm{ORF}$ is critical for

D. Wang and X. Zhang are co-first authors.

${ }^{\dagger}$ Corresponding author: J. Ye; Email: jianye@im.ac.cn

Funding: This work was supported by the National Science Foundation of China (31830073 and 31672001), the National Key Research and Development Program (2018YFD0201500), and the Chinese Postdoctoral Science Foundation (2018M641509).

*The $\boldsymbol{e}$-Xtra logo stands for "electronic extra" and indicates that three supplementary figures and four supplementary tables are published online.

The author(s) declare no conflict of interest.

(c) 2020 The American Phytopathological Society viral replication within the infected plant cells (Ruhel and Chakraborty 2019). Rep protein is the only indispensable protein for viral replication in planta. This multifunctional Rep protein is essential for viral DNA replication initiation, elongation, and termination through interacting with host proteins and other viral proteins (Rizvi et al. 2015). It shares significant similarity with the replication initiator proteins of eubacterial plasmids instead of known polymerases (Ruhel and Chakraborty 2019). Rep protein also plays multifunctional roles in stimulation of viral transcription and functions as a suppressor of gene silencing (Hanley-Bowdoin et al. 1999; Ramesh et al. 2017), though the interconnections among these functions are still unclear.

Various domains of Rep protein in Geminivirus spp. have been identified based on their corresponding functions. The amino terminus ( $\mathrm{N}$ terminus, amino acids 1 to 120 ) contains motifs I, II, and III, which are involved in DNA binding and cleavage. The middle oligomerization domain (amino acids 121 to 180) is involved in self-interaction and interaction with host factors. Thus far, the carboxyl-terminus (C-terminus) domain (amino acids 181 to 359) contains four known motifs (Walker A, Walker B, B' motif, and motif C), which display ATPase and helicase activities (George et al. 2014; Rizvi et al. 2015; Ruhel and Chakraborty 2019). In addition to the function of Rep protein, the nuclear magnetic resonance structure has studied for the $\mathrm{N}$ terminus of tomato yellow leaf curl virus Rep protein (Campos-Olivas et al. 2002), whereas the information about the most $C$ terminus of Rep protein (behind motif C, 305th amino acid) was not available. Also, rarely, detailed functional analysis of this fragment has been performed to understand the function related to viral virulence, replication, and interaction with the host plant.

Species of genus Begomovirus, the largest genus of plant viruses recorded to date, infect many major crops such as cassava, tomato, and cotton. The diseases caused by begomovirus infection pose a huge threat to crop plantations worldwide. There are monopartite or bipartite genomes of begomovirus. Bipartite genomes consist of DNA-A and DNA-B strands, whereas monopartite only have a DNA-A strand, sometimes accompanied by a satellite strand (Islam et al. 2018). Cassava mosaic viruses (CMVs), a group of bipartite begomoviruses, cause the major viral diseases for the staple food crop-cassava (Manihot esculenta Crantz) in tropical regions (Raji et al. 2016). To date, CMVs have spread to nearly all cassava-cultivating areas (Africa, South Asia, and Southeast Asia) and even infected other biodiesel crops such as Jatropha spp. (Gao et al. 2010; McCallum et al. 2017; Wang et al. 2014). Different species of CMVs refer to their first outbreak regions and are known as African cassava mosaic virus, East African cassava mosaic virus, Indian cassava mosaic virus, Sri Lankan cassava mosaic 
virus, and other variants (Hong et al. 1993; Ndunguru et al. 2005; Rey and Vanderschuren 2017).

The infection of CMVs trigger plant defense responses such as RNA silencing and hormonal signaling pathways to counter virus infection. Defensive phytohormones such as salicylic acid (SA), jasmonic acid (JA), and ethylene (ET) have been known to regulate virus resistance (Alazem and Lin 2015; Collum and Culver 2016). Among them, SA has always been studied extensively because it is essential for the establishment of both basal defense and systemic acquired resistance against viruses. Activation of SA signaling triggers plant responses such as expression of pathogenesis-related $(P R)$ genes, reactive oxygen species (ROS) burst, and hypersensitive response (HR) (Boatwright and Pajerowska-Mukhtar 2013; Carr et al. 2019; Fu and Dong. 2013; Torres et al. 2006; Vlot et al. 2009).

Sri Lankan cassava mosaic virus (SLCMV) was identified as causing the first cassava mosaic disease in Southeast Asia in 2015. In 2018, we reported a Chinese strain of SLCMV (SLCMV-HN7) from multiple plantations in Hainan and Fujian Provinces; that strain shared the highest sequence similarity with SLCMV Cambodia strain identified in 2015 (Wang et al. 2016; Wang et al. 2018). In this study, we characterized the second SLCMV strain identified from cassava plants in China. This second SLCMV strain showed $99.9 \%$ DNA-A sequence identity with the existing member of SLCMV, the Colombo strain (SLCMV-Col) (Saunders et al. 2002). The infectious clones of both SLCMV strains were generated and used to infect model plants Nicotiana benthamiana and $N$. tabacum. The virulence of SLCMV-Col is much stronger than SLCMV-HN7, indicated by the higher virus titer, more severe symptoms, and higher level of host defensive responses. The possible mechanism of the virulence differentiation for SLCMV strains was explored. The 7-amino-acid motif of Rep protein was found to be involved in virulence determination and might also trigger SA signaling against SLCMV infection in N. benthamiana.

\section{RESULTS}

Identification of two SLCMV strains from cassava plantations in China.

During the same field sample analysis in 2018, we identified another SLCMV strain. The whole-genome sequence analysis on the second Chinese strain characterized it as almost identical to that of the typical Sri Lankan Colombo strain (designed as SLCMV-Col), which was the founder virus of this species by Saunders et al. (2002) from cassava plants.

Comparative genomic analysis of these two SLCMV strains identified $93.1 \%$ nucleotide sequence identity of the DNA-A component between SLCMV-HN7 and SLCMV-Col (Supplementary Table S1). We further analyzed the amino acid sequence identity of the eight proteins encoded by DNA-A and DNA-B components of these two strains. The DNA-A component encodes six proteins known as AV1, AV2, AC1, AC2, $\mathrm{AC} 3$, and AC4, and the amino acid sequence identity of these six proteins was 96.5, 84.9, 96, 95.6, 89.6, and 95\%, respectively. The DNA-B component encodes two proteins known as BV1 and BC1, and the amino acid sequence identity of these two proteins was 81.8 and $94.1 \%$, respectively. These results indicate that two strains of SLCMV encode proteins with various sequence identities.

\section{Higher virulence of strain SLCMV-Col than that of strain SLCMV-HN7.}

To understand the relationships between genome sequence and virulence of two SLCMV strains, we next generated infectious clones of DNA-A and DNA-B for these two strains. The infectious clones of these two SLCMV strains were agroinfiltrated into leaves of 2- to 3-week-old $N$. benthamiana plants. Strain SLCMV-Col showed much stronger virulence in $N$. benthamiana (Fig. 1). At 7 days postinoculation (dpi), plants inoculated with DNA-A and DNA-B of SLCMV-Col all showed leaf yellowgreen mosaic and leaf curling symptoms. However, no obvious disease symptoms could be observed in the plants inoculated with DNA-A and DNA-B of SLCMV-HN7. At 11 dpi, SLCMVHN7-infected plants began showing weak disease symptoms such as leaf curling and mosaic, and subsequently progressed into a later stage of much more severe disease symptoms at $21 \mathrm{dpi}$ (Fig. 1A). We performed quantitative PCR (qPCR) to detect the virus accumulation level in $N$. benthamiana plants infected with two virus strains. In agreement with the much higher virulence of the strain SLCMV-Col, the viral DNA accumulated into much higher levels during the whole disease process.
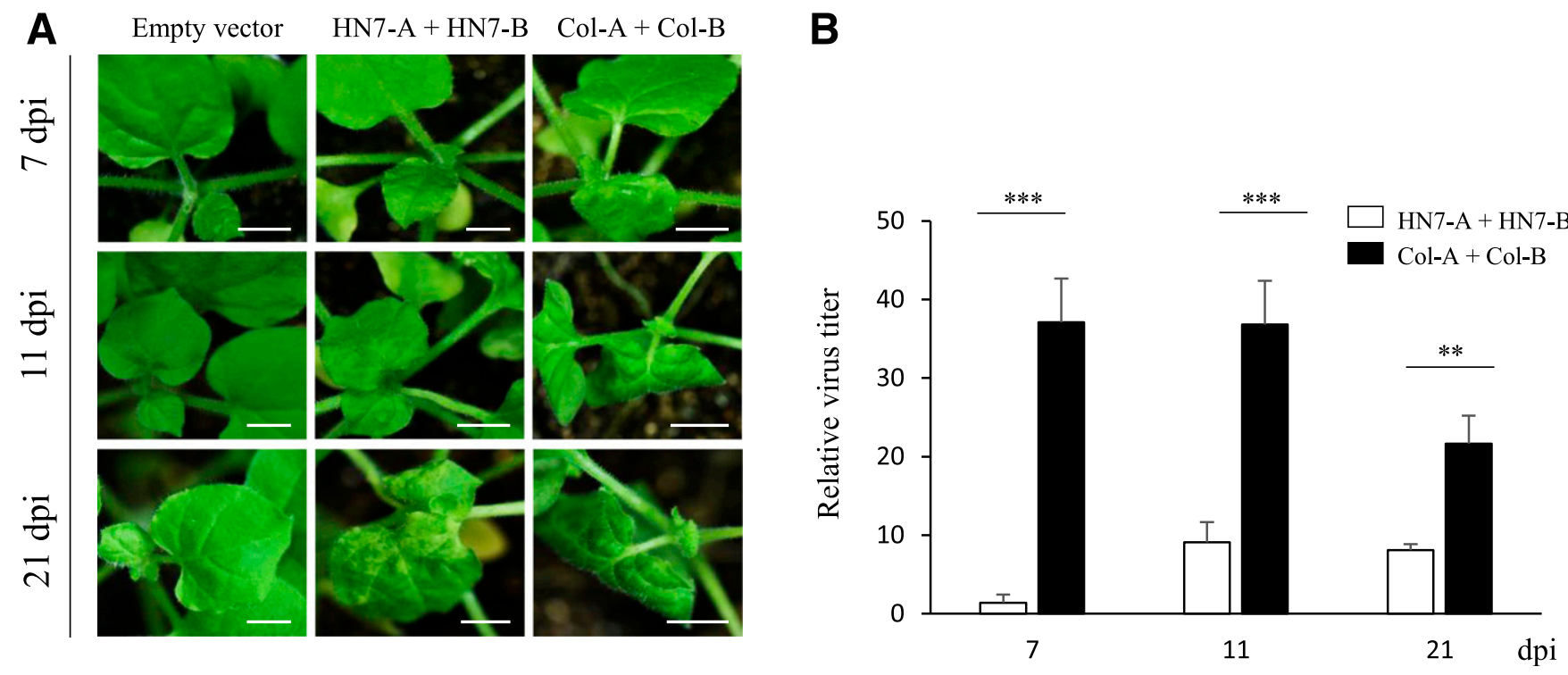

Fig. 1. Virulence of Sri Lankan cassava mosaic virus (SLCMV)-HN7 is weaker than SLCMV-Col in Nicotiana benthamiana. A, Symptoms of N. benthamiana inoculated with Agrobacterium harboring empty vector, HN7 DNA-A and DNA-B, and Col DNA-A and DNA-B at 7 days postinoculation (dpi) (upper panel), $11 \mathrm{dpi}$ (middle panel), and $21 \mathrm{dpi}$ (bottom panel). Bar $=1 \mathrm{~cm}$. B, Relative virus titer of $N$. benthamiana inoculated with Agrobacterium containing HN7 DNA-A and DNA-B, and Col DNA-A and DNA-B at 7, 11, and 21 dpi. Values are mean + standard deviation $(n=4)$. The experiment was repeated three times and obtained the same result (Student's $t$-test; *, **, and *** indicate $P<0.05,0.01$, and 0.001 , respectively). 
In addition to $N$. benthamiana, we also observed a higher virulence and a higher infectivity of SLCMV-Col than that of strain SLCMV-HN7 in another host plant, N. tabacum (Supplementary Fig. S1). In addition to different onset times of these two viruses on $N$. tabacum, their systemic infection rates were also different. The systemic infection rate of strain SLCMV-Col was $100 \%$ whereas the systemic infection rate of SLCMV-HN7 was only $20 \%$ (Supplementary Table S2). These results illustrate that SLCMV-HN7 acts as a mild stain compared with SLCMV-Col.

\section{SLCMV-Col triggers stronger host defense responses than those of strain SLCMV-HN7.}

According to the different virulence of SLCMV-HN7 and SLCMV-Col, we next asked whether the defense response in plants had different patterns. We assayed the gene expression levels of different hormone signaling pathways such as the SArelated gene PRla, the JA synthesis-related gene lipoxygenase $(L O X)$, and the ET-related gene ethylene responsive factor 1 (ERF1) (Wu et al. 2017). The gene expressions of LOX, ERF1, and PRIa were all upregulated in $N$. benthamiana inoculated with two virus strains compared with those of empty-vectorinfiltrated plants (Fig. 2A). Notably, the expression level of $P R l a$, the SA marker gene, was significantly upregulated in
SLCMV-Col-inoculated $N$. benthamiana compared with that of SLCMV-HN7. This indicated that the host SA-related pathway might be involved in the SLCMV-host interaction. To test this hypothesis, we further detected the expression levels of other genes involved in the SA pathway. As expected, the expression levels of nonexpresser of PRI (NPRI) and PR5 were also remarkably upregulated in SLCMV-Col-inoculated $N$. benthamiana compared with that of SLCMV-HN7 (Fig. 2A). The result indicates that the SA-related pathway plays an important role in response to these two different virulence strains of SLCMV.

ROS burst is a basic strategy of plant to cope with pathogen infection, which can induce HR and lead to plant necrosis (Rojas and Mysore 2012; Rojas et al. 2012). We assayed the expression levels of ROS related genes. The respiratory burst oxidase $(R B O H)$ genes $R B O H A$ and $R B O H B$ were expressed significantly higher in SLCMV-Col-inoculated than SLCMV-HN7-inoculated $N$. benthamiana (Fig. 2B), whereas no difference in glycolate oxidase $(G O X)$ gene expression was detected in plants treated with the empty vector and both SLCMV strains. This revealed that RBOHA and $R B O H B$ but not $G O X$ were involved in ROS burst in $N$. benthamiana plants for SLCMV virulence. Because $R B O H$ functions in catalyzing $\mathrm{O}^{2}$ to generate $\mathrm{O}^{2-}$, we conducted $3,3^{\prime}$-diaminobenzidine
A

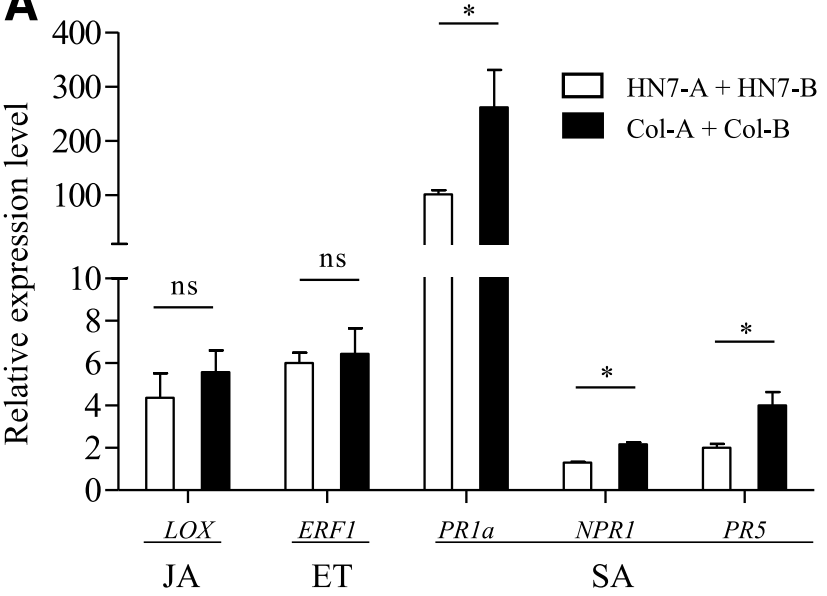

B

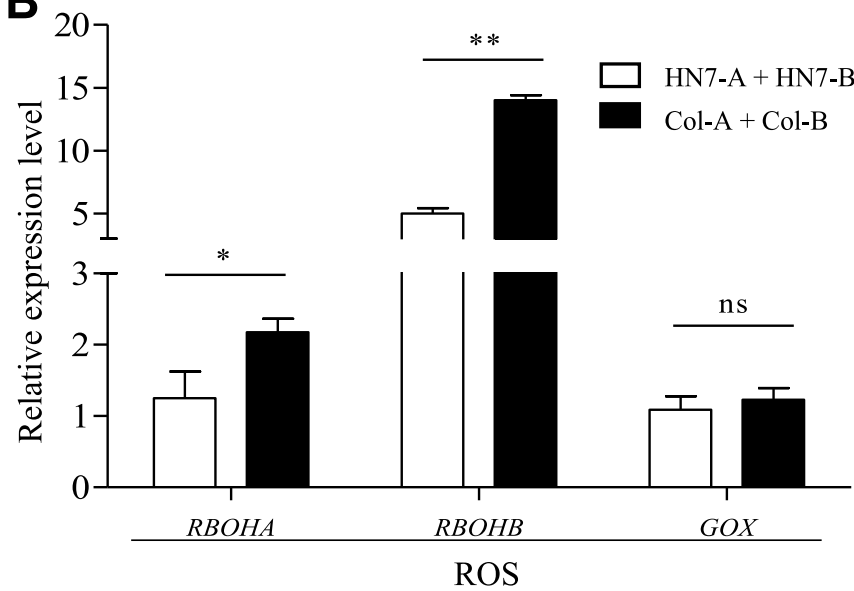

$\mathbf{E}$

E
35 dpi

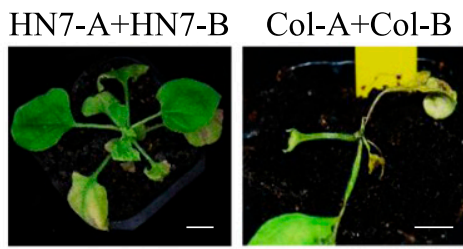

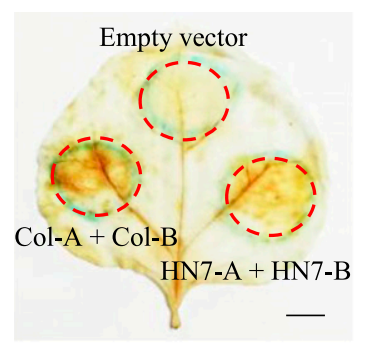

D Empty vector

$28 \mathrm{dpi}$

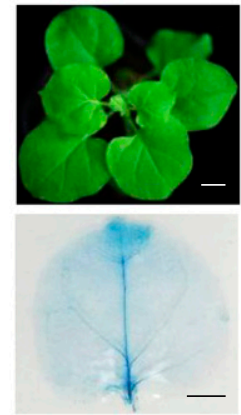

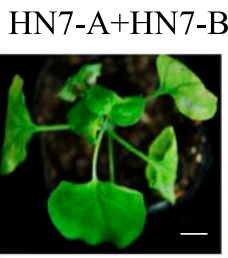
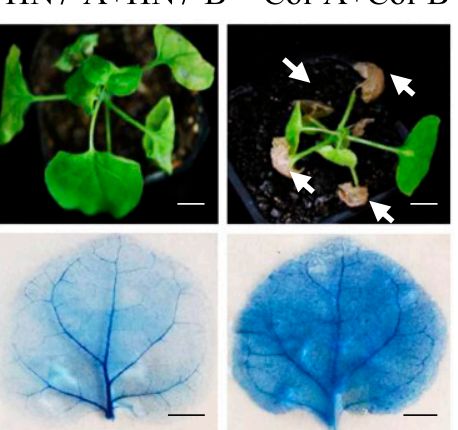

Fig. 2. Plant defense responses triggered by Sri Lankan cassava mosaic virus (SLCMV)-HN7 and SLCMV-Col in Nicotiana benthamiana. A, Relative expression level of maker genes of different hormone signaling pathways in $N$. benthamiana inoculated with empty vector, HN7 DNA-A and DNA-B, and Col DNA-A and DNA-B at 7 days postinoculation (dpi). JA = jasmonic acid, ET = ethylene, and SA = salicylic acid. B, Relative expression level of genes involved in reactive oxygen species (ROS) response at $7 \mathrm{dpi}$. (Student's $t$-test; *,**, and *** indicate $P<0.05,0.01$, and 0.001 , respectively). Values are mean + standard deviation $(n=4)$. C, 3,3'-Diaminobenzidine tetrahydrochloride staining of an $N$. benthamiana leaf infiltrated with Agrobacterium containing empty vector, HN7 DNA-A and DNA-B, and Col DNA-A and DNA-B at 3 dpi. D, Disease symptoms (upper panel) and trypan blue staining (bottom panel) of $N$. benthamiana inoculated with HN7 DNA-A and DNA-B, and Col DNA-A and DNA-B at 28 dpi. Bar $=1 \mathrm{~cm}$. E, Disease symptoms of $N$. benthamiana inoculated with HN7 DNA-A and DNA-B, and Col DNA-A and DNA-B at 35 dpi. Bar $=1 \mathrm{~cm}$. 
tetrahydrochloride (DAB) staining to detect ROS levels in $N$. benthamiana inoculated with these two virus strains. The results displayed an obvious ROS burst in the SLCMV-Colinoculated area compared with that of SLCMV-HN7 on the same leaf (Fig. 2C). During the whole inoculation stage, SLCMV-Col-inoculated $N$. benthamiana but not SLCMV-HN7 showed necrotic leaves at $28 \mathrm{dpi}$. This illustrated that SLCMVCol triggered a much stronger SA-involved defense and ROS accumulation, which may explain the observed necrosis in the infected leaves of $N$. benthamiana. That was more evident in trypan blue staining experiments, which showed more necrotic cells in SLCMV-Col-inoculated $N$. benthamiana compared with SLCMV-HN7-inoculated $N$. benthamiana (Fig. 2D), and the whole plant inoculated with SLCMV-Col was wilted at 35 dpi (Fig. 2E). The above results show that an intense defense response in plants is triggered by SLCMVCol, the higher-virulence strain.
DNA-A is critical for virulence determination of SLCMV.

In order to explore which component plays an important role in viral virulence between these two SLCMV strains, we exchanged the DNA-A and DNA-B components of SLCMVHN7 and SLCMV-Col and examined the virulence of these two pseudorecombinants. At $7 \mathrm{dpi}$, newly emerging leaves of $N$. benthamiana inoculated with SLCMV-Col DNA-A and SLCMV-HN7 DNA-B displayed disease symptoms like those of wild-type (WT) SLCMV-Col DNA-A and DNA-B, the pseudorecombinant of SLCMV-HN7 DNA-A and SLCMVCol DNA-B caused the same symptoms at $11 \mathrm{dpi}$, also resembling WT SLCMV-HN7 DNA-A and DNA-B. At 21 dpi, disease symptoms of plants inoculated with these two pseudorecombinants continued to develop (Fig. 3A). We also detected the relative virus titer of these two pseudorecombinant-inoculated plants, and results showed that the viral accumulation of plants inoculated by SLCMV-Col

B

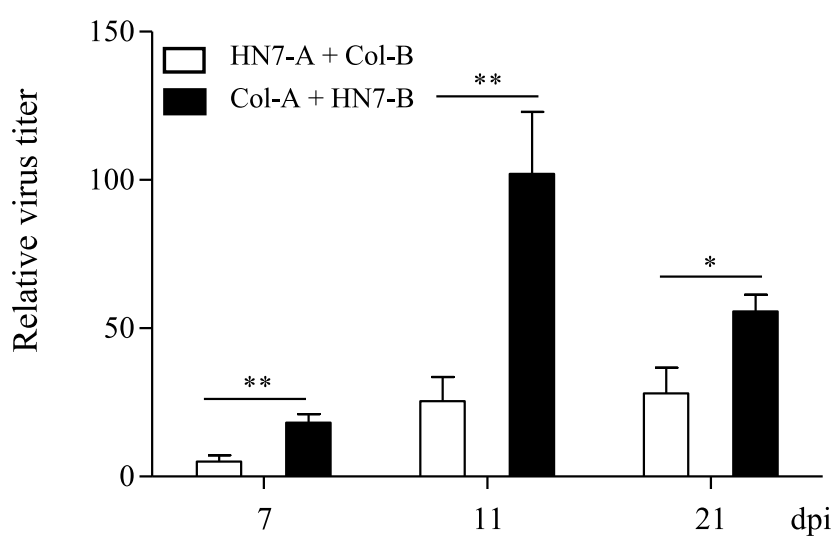

C

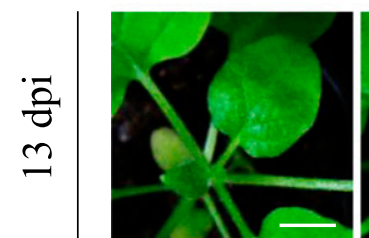

Empty vector
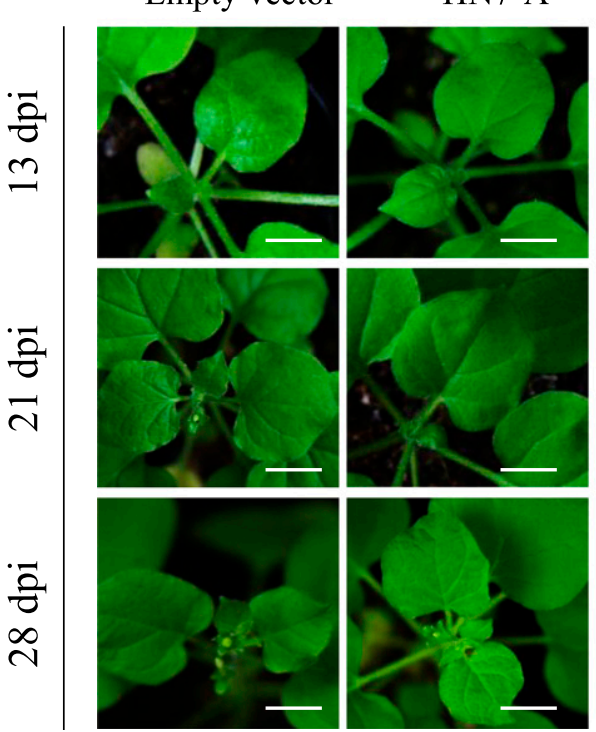

Col-A + HN7-B

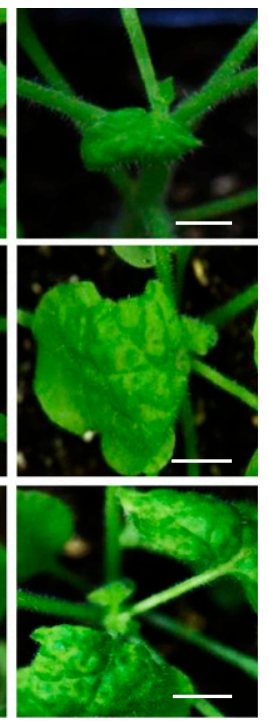

Col-A
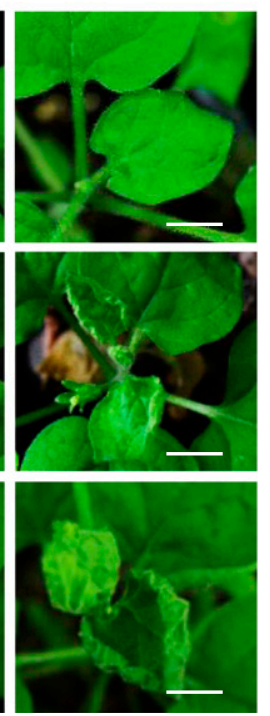

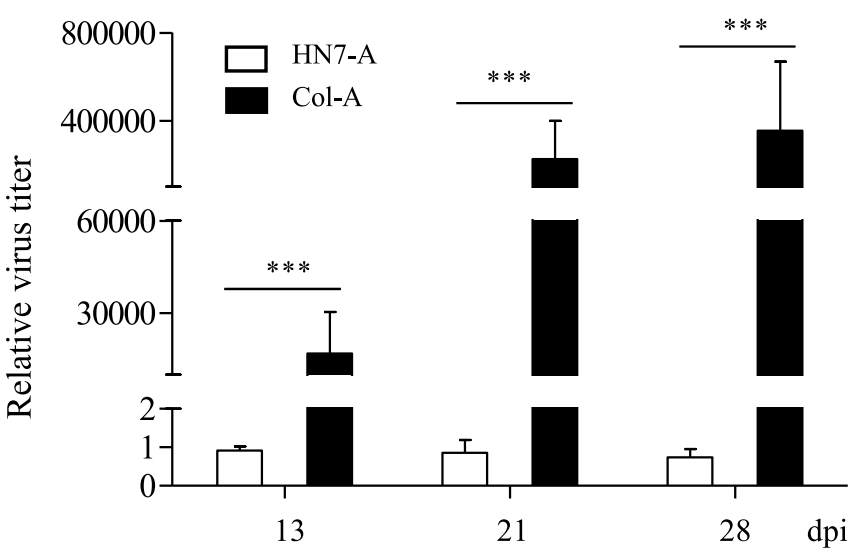

Fig. 3. DNA-A is crucial for viral virulence of Sri Lankan cassava mosaic virus (SLCMV). A and B, Disease symptoms and relative virus titer of Nicotiana benthamiana inoculated with Agrobacterium containing empty vector, HN7 DNA-A and Col DNA-B, and Col DNA-A and HN7 DNA-B at 7, 11, and 21 days postinoculation (dpi). C and D, Disease symptoms and relative virus titer of $N$. benthamiana inoculated with Agrobacterium containing empty vector, HN7 DNA-A, and Col DNA-A at 13, 21, and $28 \mathrm{dpi}$. In A and C, bar $=1 \mathrm{~cm}$. In B and D, values are mean + standard deviation $(n=4)$. (Student's $t$-test; *, **, and $* * *$ indicate $P<0.05,0.01$, and 0.001 , respectively). 
DNA-A and SLCMV-HN7 DNA-B was higher than that of plants inoculated by SLCMV-HN7 DNA-A and SLCMV-Col DNA-B at different stages (Fig. 3B). These results indicate that the different virulence of SLCMV-Col and SLCMV-HN7 in N. benthamiana is largely due to their DNA-A components.

To confirm this result further, we also inoculated these two pseudorecombinants into $N$. tabacum. Analogously, a higher virulence of SLCMV-Col DNA-A and SLCMV-HN7 DNA-B was observed than that of SLCMV-HN7 DNA-A and SLCMVCol DNA-B in N. tabacum. (Supplementary Fig. S2). Moreover, compared with WT SLCMV-HN7, the systemic infection rate of SLCMV-Col DNA-A- and SLCMV-HN7 DNA-Binoculated $N$. tabacum was increased to $100 \%$, whereas the systemic infection rate of $N$. tabacum inoculated with SLCMVHN7 DNA-A and SLCMV-Col DNA-B was decreased to 30\% compared with WT SLCMV-Col (Supplementary Table S3). These results confirm that the DNA-A component plays an important role in viral virulence in $N$. tabacum.

As reported previously, the DNA-A component of SLCMVCol has the monopartite begomovirus characteristics because the single DNA-A component can move systematically and cause virus-infected symptom in $N$. benthamiana (Saunders et al. 2002). We infiltrated the single DNA-A component of two SLCMV strains individually into $N$. benthamiana. At 13 dpi, the newly emerging leaves of SLCMV-Col DNA-A-inoculated $N$. benthamiana appeared to have a slightly upward-curling symptom and the symptom was developed more severely at 21 and $28 \mathrm{dpi}$; however, the SLCMV-HN7 DNA-A-inoculated $N$. benthamiana plants had no obvious disease symptom until 28 dpi (Fig. 3C). We detected the accumulation of DNA-A in systemic leaves of SLCMV-HN7- and SLCMV-Col-inoculated plants at 13,21 , and $28 \mathrm{dpi}$. The result showed continuous accumulation of SLCMV-Col DNA-A but we failed to detect SLCMV-HN7 DNA-A accumulation at these three stages (Fig. 3D). Taken together, these results suggest that SLCMV-Col has much stronger virulence than SLCMV-HN7, and the SLCMV DNA-A component determines viral virulence.

\section{The 7-amino-acid motif at the most $\mathrm{C}$ terminus of Rep protein contributes to viral accumulation and virulence.}

We next asked which protein encoded in DNA-A affects SLCMV virulence. We noticed that there was a 7-amino-acid deletion at the $\mathrm{C}$ terminus of Rep protein of SLCMV-HN7 $\left(\operatorname{Rep}_{\mathrm{HN} 7}\right)$ due to a $\mathrm{C} \rightarrow \mathrm{T}$ point mutation, which generated a premature protein translation termination of Rep protein but did not affect the overlapped AC2 amino acid sequence (Fig. 4A). We compared Rep protein sequences of all reported CMVs and found that $\operatorname{Rep}_{\mathrm{HN} 7}$ is the shortest one. More interestingly, Rep proteins of SLCMV after the first identification in Southeast Asia (Cambodia strain) all lack this 7-amino-acid motif (Supplementary Fig. S3). These results suggested a possible role of this 7-amino-acid motif for the current SLCMV epidemic in these countries. To test the significance of this motif, we generated a gain-of-function mutant, SLCMV HN7-A ${ }^{+7}$, in which a $\mathrm{T} \rightarrow \mathrm{C}$ mutation was introduced to mimic Rep protein of SLCMV-Col. We also generated a loss-of-function mutation, SLCMV Col- $\mathrm{A}^{-7}$, in which a $\mathrm{C} \rightarrow \mathrm{T}$ mutation was introduced in SLCMV Col DNA-A without affecting the overlapped AC2 protein sequence. We inoculated WT SLCMV Col-A and lossof-function SLCMV Col- $\mathrm{A}^{-7}$ in N. benthamiana to detect their virulence and virus accumulation. At $13 \mathrm{dpi}$, the symptoms induced by DNA-A of SLCMV Col-A ${ }^{-7}$ were much milder compared with those of SLCMV Col-A (Fig. 4B). Moreover, the virus accumulation of SLCMV Col- $\mathrm{A}^{-7}$ was reduced to $50 \%$ compared with SLCMV Col-A-infected systemic leaves (Fig. 4C). Considering the fact that SLCMV HN7-A could not produce systemic movement, we examined the accumulation of
SLCMV HN7-A and SLCMV HN7-A ${ }^{+7}$ in the inoculated leaves. At $1 \mathrm{dpi}$, the accumulation levels between SLCMV HN7-A and SLCMV HN7-A ${ }^{+7}$ showed no significant difference whereas, at 2 and $3 \mathrm{dpi}$, the accumulation of SLCMV HN7-A ${ }^{+7}$ was significantly increased compared with that of WT SLCMV HN7-A (Fig. 4D). These results verify that the 7amino-acid motif at the $\mathrm{C}$ terminus of Rep protein is important for the virulence determination of SLCMV in N. benthamiana.

\section{The 7-amino-acid motif of Rep protein enhances protein accumulation and triggers stronger host defensive response.}

Because we observed an intense defense response in $N$. benthamiana against SLCMV, we wondered whether the Rep protein of SLCMV is involved in triggering a host defense response. Therefore, we detected the gene expression in the SA pathway by transient expression of $\operatorname{Rep}_{\mathrm{Col}}$ and Rep $\mathrm{HN}_{\mathrm{HN}}$. Compared with Rep $\mathrm{HN}_{\mathrm{HN}}$, much higher NPRl and PRla expression levels were induced by Rep $_{\text {Col }}$ transiently expressed $N$. benthamiana (Fig. 4E). This result suggests that Rep $\mathrm{Col}_{\text {, }}$, which contains the 7-amino-acid motif, was critical for triggering host defense against SLCMV.

To investigate the possible biological function of the 7amino-acid motif of Rep protein, we examined the protein accumulation level of Rep protein in plants. We found that the protein accumulation of Rep $\mathrm{Col}_{\text {was }} 2.18$-fold that of Rep $\mathrm{p}_{\mathrm{HN} 7}$ (Fig. 4F) but the mRNA expression of $\mathrm{Rep}_{\mathrm{Col}}$ and Rep $\mathrm{p}_{\mathrm{HN}}$ showed no obvious difference (Fig. 4G), which indicated that the 7-amino-acid motif of SLCMV Rep protein might play an important role in the protein accumulation in plants. To further establish this hypothesis, we constructed gain-of-function mutation $\operatorname{Rep}_{\mathrm{HN} 7}{ }^{+7}$ and loss-of-function mutation $\mathrm{Rep}_{\mathrm{Col}}{ }^{-7}$. Next, we compared individual protein accumulation levels at 2 dpi. Results showed that the accumulation of $\operatorname{Rep}_{\mathrm{HN} 7}{ }^{+7}$ was 1.75 -fold that of WT Rep $\mathrm{H}_{\mathrm{HN} 7}$, whereas $\operatorname{Rep}_{\mathrm{Col}}{ }^{-7}$ was only ap-

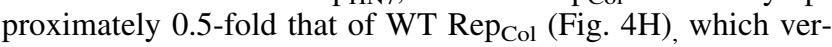
ified that the 7-amino-acid motif was important for Rep protein accumulation in plants.

Because 26s proteasome-mediated protein degradation is an essential pathway for protein degradation (Marshall and Vierstra 2019), we investigated whether this pathway accounts for the different accumulation of $\operatorname{Rep}_{\mathrm{Col}}$ and $\mathrm{Rep}_{\mathrm{HN} 7}$ in vivo. The fact that we found no significant difference of Rep protein accumulation with or without MG132-treatment suggested that 26 s proteasome has a minor role in Rep protein degradation.

\section{DISCUSSION}

Here, we reported a comparative genomic analysis of two SLCMV strains identified from China. SLCMV-Col DNA-A has biological characteristics of a typical monopartite begomovirus, unlike that of the DNA-A of SLCMV-HN7. We functionally identified a 7-amino-acid motif of Rep protein involved in virulence determination and interaction with host SA signaling.

Phytohormones are essential for plants to counter virus infection directly or indirectly. Many reports illustrated the significance of SA, JA, and ET signaling in defending plants from DNA or RNA viruses (Alazem and Lin 2015; Collum and Culver 2016; Li et al. 2015). Especially, geminivirus invasion in a host can induce marker gene expression in the SA pathway; for example, beet severe curly top virus could induce SA marker gene $P R 5$ expression, and tomato yellow leaf curl virus could trigger PRla expression (Chen et al. 2010; Guo et al. 2016). These were consistent with what we observed in our study (Fig. 2A). Increasing evidence reveals that the plant JA pathway plays an important role in whitefly-mediated 
geminivirus transmission. For transmission of plant viruses, they evolved various strategies to attenuated JA signaling to benefit their vector ( $\mathrm{Li}$ et al. 2014; Wu et al. 2019; Zhao et al. 2019). What's more, the antagonism of SA- or JA-mediated plant defense was also important for virus-vector-host mutualistic interactions. For example, tomato spotted wilt virus could induce the SA pathway but inhibit the JA pathway to abolish the SA-JA antagonism and finally benefit its vector,

A

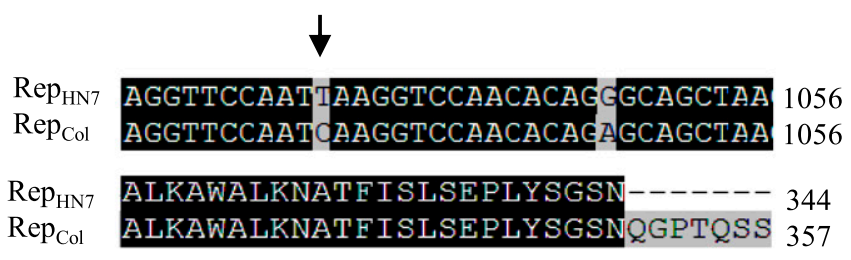

thrips (Abe et al. 2012). In our study, the marker gene in the JA pathway showed no obvious differences between SLCMVHN7- and SLCMV-Col-infected plants but the marker genes in the SA pathway in SLCMV-HN7-infected plants were remarkably decreased compared with SLCMV-Col-infected plants (Fig. 2A). These results indicated that, compared with the ancient SLCMV-Col strain, the new emerging strain SLCMV-HN7 induced attenuated host resistance response but

B

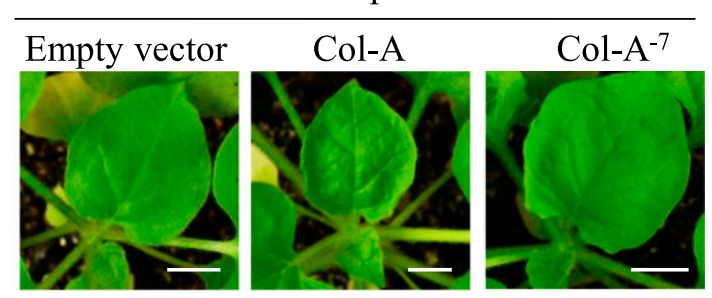

C

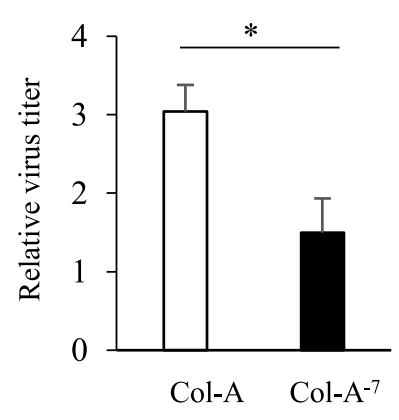

$\mathbf{F}$

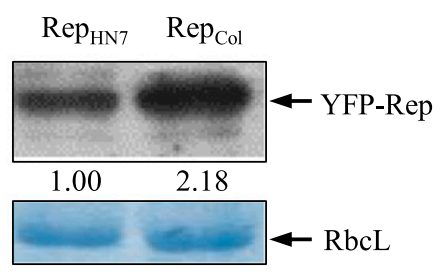

H

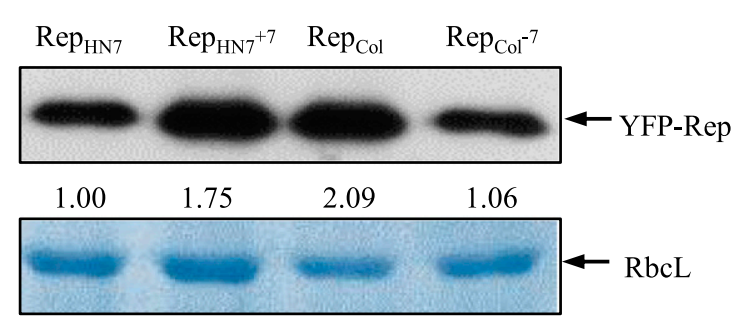

Inoculated leaf

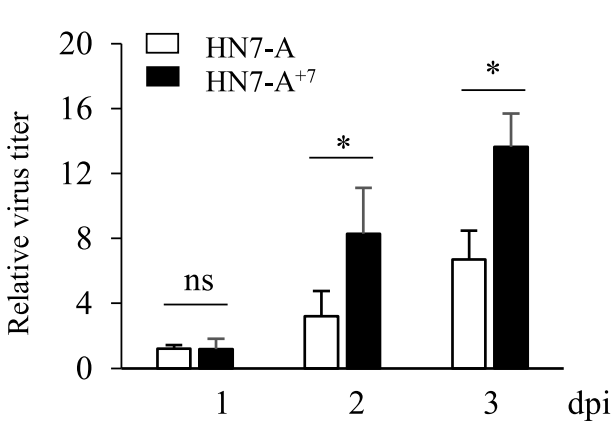

E

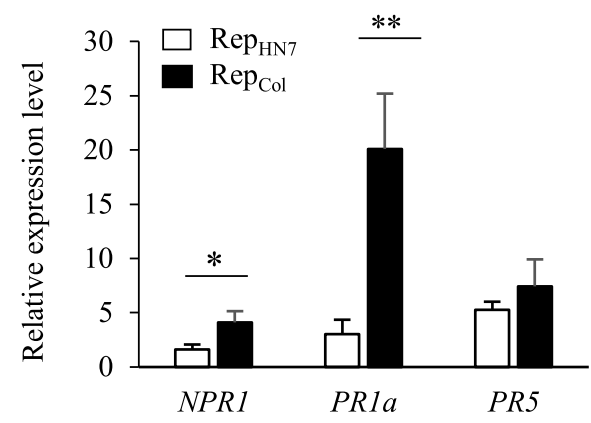

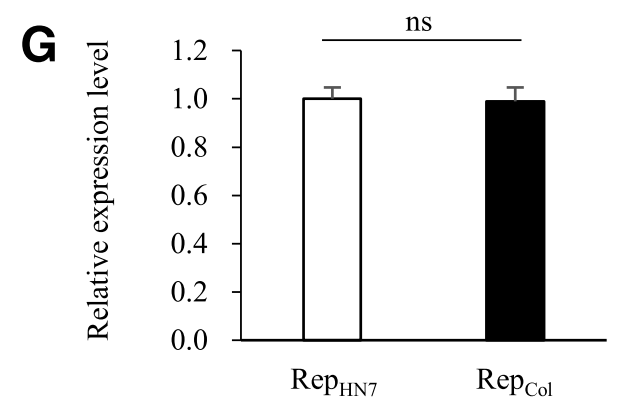

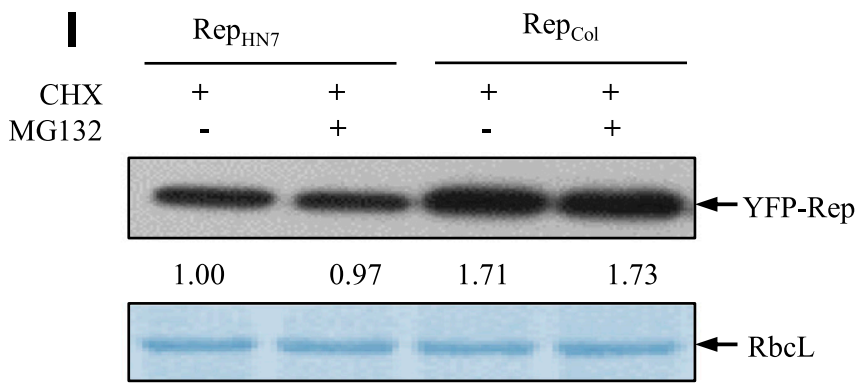

Fig. 4. The 7-amino-acid motif of replication (Rep) protein increases its protein accumulation and triggers stronger host defensive responses. A, Sequence alignment of nucleotide acids and amino acids between $R_{\text {epol }}$ and Rep $\mathrm{P}_{\mathrm{HN} 7}$. Black arrow indicates the nucleotide acid site which generated a premature termination of Rep $\mathrm{HN}_{7}$. B, Symptom of upward-curling leaf was more attenuated on Nicotiana benthamiana inoculated with Col- $\mathrm{A}^{-7}$ than Col-A. Bar $=1 \mathrm{~cm}$; dpi $=$ days postinoculation. C, Relative virus titer of systemic leaves of $N$. benthamiana inoculated with Col-A and Col- $\mathrm{A}^{-7}$ at 13 dpi. D, Relative virus titer of inoculated leaves of $N$. benthamiana infiltrated with HN7-A and HN7- $\mathrm{A}^{+7}$ at 1,2 , and 3 dpi. E, Gene expression levels of $N$. benthamiana transiently expressed with $\mathrm{Rep}_{\mathrm{Col}}$ and $\mathrm{Rep}_{\mathrm{HN} 7}$ at 2 dpi. F, Protein accumulation of Rep $\mathrm{Col}_{\mathrm{Col}}$ and $\mathrm{Rep}_{\mathrm{HN} 7}$ in N. benthamiana at 2 dpi. G, Relative expression levels of Rep $\mathrm{HN}_{\mathrm{HN}}$ and Rep $\mathrm{Rol}_{\mathrm{Col}}$ mRNA at 2 dpi. H, Protein accumulation of Rep $\mathrm{HN}_{7}$ and $\operatorname{Rep}_{\mathrm{HN} 7}{ }^{+7}$ as well as Rep $\mathrm{Col}$ and $\operatorname{Rep}_{\mathrm{Col}^{-1}}{ }^{-7}$ in N. benthamiana at 2 dpi. I, Effect of MG132 on the protein

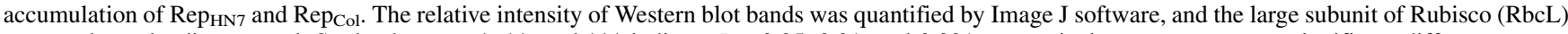
was used as a loading control. Student's $t$ test; *, **, and $* * *$ indicate $P<0.05,0.01$, and 0.001 , respectively; ns represents no significant differences. 
did not affect the feeding behavior of its vectors. Moreover, the virulence of a virus can largely benefit from Rep-proteinmediated virus replication in the host (Rizvi et al. 2015). In the current study, the 7-amino-acid of Rep protein which played an important role in virus accumulation mostly existed in CMVs discovered before 2015, which illustrated that the 7 amino acids might be lost during virus evolution. Most importantly, because of its mild virulence, SLCMV-HN7 usually produces no obvious disease symptom in cassava; thus, the virus-infected cassava is free from being removed, which can keep the virus existent latently (Minato et al. 2019). Taken together, in an evolutionary perspective, these characteristics of SLCMV-HN7 (low virulence, decreased accumulation capacity, and inducing a weakened host defense response) might be a benefit for its prevalence and transmission in the long term.

Protein accumulation in plants is determined by transcription, synthesis, and degradation. In our study, similar expression levels of $\operatorname{Rep}_{\mathrm{Col}}$ and Rep $\mathrm{HN}_{\mathrm{HN}} \mathrm{mRNA}$ indicated that different Rep protein accumulation was not due to the transcription stage (Fig. 4G). Likewise, 26s proteasome was not involved in the degradation of Rep protein because the protein accumulation was no different in leaves treated with or without MG132 (Fig. 4I). Therefore, the different protein accumulation of $\operatorname{Rep}_{\mathrm{Col}}$ and $\mathrm{Rep}_{\mathrm{HN} 7}$ might due to a protein synthesis pathway or other protein degradation pathways, such as autophagy, because many studies reported that geminiviral proteins are targeted by autophagy-related proteins for degradation. For example, the virulence factor $\beta C 1$ of cotton leaf curl Multan virus is targeted by autophagy-related protein ATG8 for degradation to resist virus infection (Haxim et al. 2017). Further studies need to be conducted to verify these hypotheses.

In conclusion, we discovered two different virulence strains of SLCMV in China. The hypervirulence strain SLCMV-Col but not mild strain SLCMV-HN7 harbors a 7-amino-acid motif at the most $\mathrm{C}$ terminus of Rep protein, which is essential for viral virulence and triggered host defense. This is the first report about the function mechanism of the most $\mathrm{C}$ terminus of Rep protein, which provides further information about the role of Rep protein.

\section{MATERIALS AND METHODS}

\section{Plant materials and growth conditions.}

$N$. benthamiana and $N$. tabacum were cultivated in a growth chamber at $25^{\circ} \mathrm{C}$ under short-day conditions $(10 \mathrm{~h}$ of light and $14 \mathrm{~h}$ of darkness).

\section{Plasmid construction.}

For the infectious clone of SLCMV-HN7, total DNA of cassava with yellow-green mosaic, curling symptoms was extracted using the cetyltrimethylammonium bromide (CTAB) method. The full length of SLCMV-HN7 DNA-A and DNA-B components were sequenced in our previous study (Wang et al. 2018). The infectious clone of SLCMV-HN7 was constructed as previously described, with little modification (Gao et al. 2010). The product of full-length SLCMV-HN7 DNA-A was added with a PstI site at each end, then digested with HindIII to produce $0.4 \mathrm{~A}$ and $0.6 \mathrm{~A}$. The $0.6 \mathrm{~A}$ was inserted into the HindIII and PstI sites of pCAMBIA1300 to produce pCAMBIA1300HN7-0.6A. After dephosphorylation, the PstI-digested pCAMBIA1300-HN7-0.6A was ligated with the 1.0A to produce the infectious clone of SLCMV-HN7 DNA-A, pCAMBIA1300-HN7-1.6A. The product of full-length SLCMV-HN7 DNA-B was added with a BamHI site at each end, then digested with HindIII to produce $0.48 \mathrm{~B}$ and $0.52 \mathrm{~B}$.
The $0.52 \mathrm{~B}$ was inserted into the HindIII and BamHI sites of pCAMBIA1300 to produce pCAMBIA1300-HN7-0.52B. After dephosphorylation, the BamHI-digested pCAMBIA1300-HN7$0.52 \mathrm{~B}$ was ligated with the 1.0B to produce a clone of SLCMVHN7 DNA-B, pCAM1300-HN7-1.52B. The infectious clones of SLCMV-Col DNA-A and DNA-B were constructed the same as those for SLCMV-HN7.

For Rep protein point mutant of SLCMV-HN7 and SLCMVCol, the full length of SLCMV-HN7 and SLCMV-Col DNA-A was cloned into pEASY-T3 cloning vector to conduct mutagenesis, and then the infectious clone was constructed as described above. All infectious clones were verified by DNA sequencing.

For protein expression vector, the genes of Rep ${ }_{\mathrm{HN} 7}$ $\operatorname{Rep}_{\mathrm{HN} 7}{ }^{+7}, \mathrm{Rep}_{\mathrm{Col}}$, and $\mathrm{Rep}_{\mathrm{Col}}{ }^{-7}$ were amplified using primer pairs Rep $_{\text {HN7 }}-$ Kpn 1-F/Rep ${ }_{\text {HN7 }}-$ Xhol-R, Rep ${ }_{\text {HN7 }}-$ Kpn 1-F/RepHN7 ${ }^{+7}$ Xhol-R, Rep $\mathrm{Col}^{-K p n 1-F / R e p} \mathrm{Col}-\mathrm{Xhol}-\mathrm{R}$, and $\mathrm{Rep}_{\mathrm{Col}}-\mathrm{Kpn} 1-$ F/Rep ${ }_{\text {HN7 }}$ Xhol-R, respectively (Supplementary Table S4). The PCR products were cloned into pENTR3C vector by a one-step clone method following the manufacturer's protocol (C112-02$\mathrm{AB}$; Vazyme), then cloned into the pH7WG2Y vector through Gateway LR reaction to generate $\mathrm{pH} 7 \mathrm{WG} 2 \mathrm{Y}-\mathrm{Rep}_{\mathrm{HN}}$, $\mathrm{pH} 7 \mathrm{WG} 2 \mathrm{Y}-$ $\mathrm{Rep}_{\mathrm{HN} 7+7}, \mathrm{pH} 7 \mathrm{WG} 2 \mathrm{Y}-\mathrm{Rep}_{\mathrm{Col}}$, and pH7WG2Y- $\mathrm{Rep}_{\mathrm{Col}^{-7} \text {. }}$.

\section{Agrobacterium infiltration.}

All of the binary vectors constructed in this study were transformed into Agrobacterium strain EHA105 by electroporation; an empty vector of pCAMBIA1300-transformed Agrobacterium strain EHA105 was used as negative control. The Agrobacterium-containing SLCMV-HN7 or SLCMV-Col infectious clones were cultivated on Luria-Bertani medium with added spectinomycin and rifampicin antibiotics at $28^{\circ} \mathrm{C}$ overnight, and the agrobacterial cells were resuspended in MMA buffer (10 mM morpholineethanesulfonic acid, $10 \mathrm{mM}$ $\mathrm{MgCl}_{2}$, and $200 \mu \mathrm{M}$ acetosyringone). Agrobacterium-harbored DNA-A or DNA-B were mixed in equal volume and the mixture was infiltrated into 2- to 3-week-old $N$. benthamiana plants.

\section{qPCR and reverse-transcription qPCR.}

Total DNA and RNA of agroinfiltrated $N$. benthamiana was extracted using CTAB and the RNeasy Plant Mini Kit (number 74904; Qiagen), respectively. Total RNA (1 $\mu \mathrm{g}$ ) for each sample was reverse transcribed using the TransScript One-Step gDNA Removal and cDNA Synthesis SuperMix (AT311-03; TRAN). Four independent biological samples, each from an independent plant, were collected and analyzed. Reverse-transcription (RT)-qPCR or qPCR was performed on the CFX 96 system (Bio-Rad) using Thunderbird SYBR qPCR mix (QPS-201; TOYOBO) (Li et al. 2014; Ye et al. 2015).

\section{DAB staining and trypan blue staining.}

Agrobacterium containing HN7 DNA-A and DNA-B and Col DNA-A and DNA-B were infiltrated into same $N$. benthamiana leaf at an optical density at $600 \mathrm{~nm}\left(\mathrm{OD}_{600}\right)=0.5$ of each Agrobacterium strain. Three days after treatment, leaves were collected and incubated with DAB solution (DAB dissolved in deionized water to a final concentration of $1 \mathrm{mg} / \mathrm{ml}$ ) overnight at room temperature in the dark; then, leaves were boiled in $95 \%$ ethanol to remove the dye. For trypan blue staining, leaves collected were immersed in trypan blue solution $(10 \mathrm{ml}$ of lactic acid, $10 \mathrm{ml}$ of $100 \%$ glycerol, $10 \mathrm{ml}$ of phenol water, $7.5 \mathrm{ml}$ of deionized water, and $0.015 \mathrm{~g}$ of trypan blue) with an equal volume ethanol, then boiled for $10 \mathrm{~min}$ and incubated at room temperature overnight. Chloral hydrate solution $(2.5 \mathrm{~g} / \mathrm{ml})$ was used to remove the dye. 


\section{Transient expression of protein assay.}

To evaluate the protein accumulation of $\mathrm{Rep}_{\mathrm{HN} 7}$ and $\mathrm{Rep}_{\mathrm{Col}}$, yellow fluorescent protein-Rep fusion protein constructs were made and transformed into Agrobacterium cells. The Agrobacterium containing pH7WG2Y-Rep ${ }_{\mathrm{HN} 7}$ and $\mathrm{pH} 7 \mathrm{WG} 2 \mathrm{Y}-$ Rep $_{\text {Col }}$ was infiltrated into each half of the same N. benthamiana leaves. The agroinfiltrated leaves were harvested at 2 dpi. Total proteins were extracted and detected by Western blot with anti-green fluorescent protein antibody (code number HT801; TransGen Biotech).

To investigate the host defense triggered by $\mathrm{Rep}_{\mathrm{HN} 7}$ and Rep $_{\mathrm{Col}}$ protein, Agrobacterium cells containing pH7WG2Y$\mathrm{Rep}_{\mathrm{HN} 7}$ or $\mathrm{pH} 7 \mathrm{WG} 2 \mathrm{Y}^{-R e p} \mathrm{P}_{\mathrm{Col}}$ were infiltrated into $\mathrm{N}$. benthamiana. The treated leaves were harvested at $3 \mathrm{dpi}$ and leaves infiltrated with Agrobacterium containing pH7WG2Y-HA vector were used as a negative control. Total RNAs were extracted and RT-qPCR was performed. All Agrobacterium cells infiltrated into $N$. benthamiana were adjusted at a concentration of $\mathrm{OD}_{600}=1.5$.

To detect the degradation of Rep $\mathrm{HN}_{\mathrm{HN}}$ and Rep $\mathrm{Pol}_{\mathrm{Cl}}$ by MG132, Agrobacterium cells containing pH7WG2Y-RepHN7 or

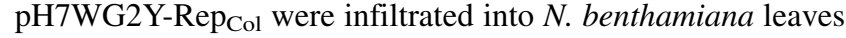
for 2 days, then infiltrated with $100 \mathrm{mM}$ cycloheximide added to $50 \mathrm{mM}$ MG132 or dimethyl sulfoxide. The infiltrated leaves were harvested at $8 \mathrm{~h}$ to detect the accumulation of $\operatorname{Rep}_{\mathrm{HN} 7}$ and Rep $_{\text {Col }}$.

\section{LITERATURE CITED}

Abe, H., Tomitaka, Y., Shimoda, T., Seo, S., Sakurai, T., Kugimiya, S., Tsuda, S., and Kobayashi, M. 2012. Antagonistic plant defense system regulated by phytohormones assists interactions among vector insect, thrips and a tospovirus. Plant Cell Physiol. 53:204-212.

Alazem, M., and Lin, N. S. 2015. Roles of plant hormones in the regulation of host-virus interactions. Mol. Plant Pathol. 16:529-540.

Boatwright, J. L., and Pajerowska-Mukhtar, K. 2013. Salicylic acid: An old hormone up to new tricks. Mol. Plant Pathol. 14:623-634.

Campos-Olivas, R., Louis, J. M., Clérot, D., Gronenborn, B., and Gronenborn, A. M. 2002. The structure of a replication initiator unites diverse aspects of nucleic acid metabolism. Proc. Natl. Acad. Sci. U.S.A. 99:10310-10315.

Carr, J. P., Murphy, A. M., Tungadi, T., and Yoon, J. Y. 2019. Plant defense signals: Players and pawns in plant-virus-vector interactions. Plant Sci. 279:87-95.

Chen, H., Zhang, Z., Teng, K., Lai, J., Zhang, Y., Huang, Y., Li, Y., Liang, L., Wang, Y., Chu, C., Guo, H., and Xie, Q. 2010. Up-regulation of LSB1/GDU3 affects geminivirus infection by activating the salicylic acid pathway. Plant J. 62:12-23.

Collum, T. D., and Culver, J. N. 2016. The impact of phytohormones on virus infection and disease. Curr. Opin. Virol. 17:25-31.

Fu, Z. Q., and Dong, X. 2013. Systemic acquired resistance: Turning local infection into global defense. Annu. Rev. Plant Biol. 64:839-863.

Gao, S., Qu, J., Chua, N. H., and Ye, J. 2010. A new strain of Indian cassava mosaic virus causes a mosaic disease in the biodiesel crop Jatropha curcas. Arch. Virol. 155:607-612.

George, B., Ruhel, R., Mazumder, M., Sharma, V. K., Jain, S. K., Gourinath, S., and Chakraborty, S. 2014. Mutational analysis of the helicase domain of a replication initiator protein reveals critical roles of

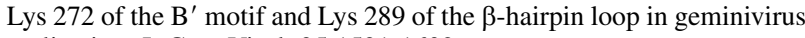
replication. J. Gen. Virol. 95:1591-1602.

Guo, H., Huang, L., Sun, Y., Guo, H., and Ge, F. 2016. The contrasting effects of elevated $\mathrm{CO}_{2}$ on TYLCV infection of tomato genotypes with and without the resistance gene, Mi-1.2. Front. Plant Sci. 7:1680.

Hanley-Bowdoin, L., Settlage, S. B., Orozco, B. M., Nagar, S., and Robertson, D. 1999. Geminiviruses: Models for plant DNA replication, transcription, and cell cycle regulation. Crit. Rev. Plant Sci. 18:71-106.

Haxim, Y., Ismayil, A., Jia, Q., Wang, Y., Zheng, X., Chen, T., Qian, L., Liu, N., Wang, Y., Han, S., Cheng, J., Qi, Y., Hong, Y., and Liu, Y. 2017. Autophagy functions as an antiviral mechanism against geminiviruses in plants. eLife 6:e23897.

Hong, Y. G., Robinson, D. J., and Harrison, B. D. 1993. Nucleotide sequence evidence for the occurrence of three distinct whiteflytransmitted geminiviruses in cassava. J. Gen. Virol. 74:2437-2443.
Islam, W., Akutse, K. S., Qasim, M., Khan, K. A., Ghramh, H. A., Idrees, A., and Latif, S. 2018. Bemisia tabaci-mediated facilitation in diversity of begomoviruses: Evidence from recent molecular studies. Microb. Pathog. 123:162-168.

Li, R., Tee, C. S., Jiang, Y. L., Jiang, X. Y., Venkatesh, P. N., Sarojam, R., and Ye, J. 2015. A terpenoid phytoalexin plays a role in basal defense of Nicotiana benthamiana against Potato virus X. Sci. Rep. 5:9682.

Li, R., Weldegergis, B. T., Li, J., Jung, C., Qu, J., Sun, Y., Qian, H., Tee, C. van Loon, J. J., Dicke, M., Chua, N. H., Liu, S. S., and Ye, J. 2014. Virulence factors of geminivirus interact with MYC2 to subvert plant resistance and promote vector performance. Plant Cell 26:4991-5008.

Marshall, R. S., and Vierstra, R. D. 2019. Dynamic regulation of the $26 \mathrm{~S}$ proteasome: From synthesis to degradation. Front. Mol. Biosci. 6:40.

McCallum, E. J., Anjanappa, R. B., and Gruissem, W. 2017. Tackling agriculturally relevant diseases in the staple crop cassava (Manihot esculenta). Curr. Opin. Plant Biol. 38:50-58.

Minato, N., Sok, S., Chen, S., Delaquis, E., Phirun, I., Le, V. X., Burra, D. D., Newby, J. C., Wyckhuys, K. A. G., and de Haan, S. 2019. Surveillance for Sri Lankan cassava mosaic virus (SLCMV) in Cambodia and Vietnam one year after its initial detection in a single plantation in 2015. PLoS One 14:e0212780.

Ndunguru, J., Legg, J. P., Aveling, T. A., Thompson, G., and Fauquet, C. M. 2005. Molecular biodiversity of cassava begomoviruses in Tanzania: Evolution of cassava geminiviruses in Africa and evidence for East Africa being a center of diversity of cassava geminiviruses. Virol. J. 2:21.

Raji, S. N., Subhash, N., Ravi, V., Saravanan, R., Mohanan, C. N., MakeshKumar, T., and Nita, S. 2016. Detection and classification of mosaic virus disease in cassava plants by proximal sensing of photochemical reflectance index. J. Indian Soc. Remote Sens. 44: $875-883$.

Ramesh, S. V., Sahu, P. P., Prasad, M., Praveen, S., and Pappu, H. R. 2017. Geminiviruses and plant hosts: A closer examination of the molecular arms race. Viruses 9:256.

Rey, C., and Vanderschuren, H. 2017. Cassava mosaic and brown streak diseases: Current perspectives and beyond. Annu. Rev. Virol. 4 429-452.

Rizvi, I., Choudhury, N. R., and Tuteja, N. 2015. Insights into the functional characteristics of geminivirus rolling-circle replication initiator protein and its interaction with host factors affecting viral DNA replication. Arch. Virol. 160:375-387.

Rojas, C. M., and Mysore, K. S. 2012. Glycolate oxidase is an alternative source for $\mathrm{H} 2 \mathrm{O} 2$ production during plant defense responses and functions independently from NADPH oxidase. Plant Signal. Behav. 7: 752-755.

Rojas, C. M., Senthil-Kumar, M., Wang, K., Ryu, C. M., Kaundal, A., and Mysore, K. S. 2012. Glycolate oxidase modulates reactive oxygen species-mediated signal transduction during nonhost resistance in Nicotiana benthamiana and Arabidopsis. Plant Cell 24:336-352.

Ruhel, R., and Chakraborty, S. 2019. Multifunctional roles of geminivirus encoded replication initiator protein. VirusDisease 30:66-73.

Saunders, K., Salim, N., Mali, V. R., Malathi, V. G., Briddon, R., Markham, P. G., and Stanley, J. 2002. Characterisation of Sri Lankan cassava mosaic virus and Indian cassava mosaic virus: Evidence for acquisition of a DNA B component by a monopartite begomovirus. Virology 293: 63-74.

Torres, M. A., Jones, J. D., and Dangl, J. L. 2006. Reactive oxygen species signaling in response to pathogens. Plant Physiol. 141:373-378.

Varsani, A., Roumagnac, P., Fuchs, M., Navas-Castillo, J., Moriones, E., Idris, A., Briddon, R. W., Rivera-Bustamante, R., Murilo Zerbini, F., and Martin, D. P. 2017. Capulavirus and Grablovirus: Two new genera in the family Geminiviridae. Arch. Virol. 162:1819-1831.

Vlot, A. C., Dempsey, D. A., and Klessig, D. F. 2009. Salicylic acid, a multifaceted hormone to combat disease. Annu. Rev. Phytopathol. 47: 177-206.

Wang, D., Yao, X. M., Huang, D. X., Shi, T., Wang, G. F., and Ye, J. 2018. First report of Sri Lankan cassava mosaic virus infected cassava in China. Plant Dis. 103:1437.

Wang, G., Sun, Y., Xu, R., Qu, J., Tee, C., Jiang, X., and Ye, J. 2014. DNAA of a highly pathogenic Indian cassava mosaic virus isolated from Jatropha curcas causes symptoms in Nicotiana benthamiana. Virus Genes 48:402-405.

Wang, H. L., Cui, X. Y., Wang, X. W., Liu, S. S., Zhang, Z. H., and Zhou, X. P. 2016. First report of Sri Lankan cassava mosaic virus infecting cassava in Cambodia. Plant Dis. 100:1029.

Wu, L., Wu, H., Chen, L., Zhang, H., and Gao, X. 2017. Induction of systemic disease resistance in Nicotiana benthamiana by the cyclodipeptides cyclo (L-Pro-L-Pro) and cyclo (D-Pro-D-Pro). Mol. Plant Pathol. 18:67-74 
Wu, X., Xu, S., Zhao, P., Zhang, X., Yao, X., Sun, Y., Fang, R., and Ye, J. 2019. The Orthotospovirus nonstructural protein NSs suppresses plant MYC-regulated jasmonate signaling leading to enhanced vector attraction and performance. PLoS Pathog. 15:e1007897.

Ye, J., Yang, J., Sun, Y., Zhao, P., Gao, S., Jung, C., Qu, J., Fang, R., and Chua, N. H. 2015. Geminivirus activates ASYMMETRIC LEAVES 2 to accelerate cytoplasmic DCP2-mediated mRNA turnover and weakens RNA Silencing in Arabidopsis. PLoS Pathog. 11:e1005196.
Zerbini, F. M., Briddon, R. W., Idris, A., Martin, D. P., Moriones, E., NavasCastillo, J., Rivera-Bustamante, R., Roumagnac, P., Varsani, A., and ICTV Report Consortium. 2017. ICTV virus taxonomy profile: Geminiviridae. J. Gen. Virol. 98:131-133.

Zhao, P., Yao, X., Cai, C., Li, R., Du, J., Sun, Y., Wang, M., Zou, Z., Wang, Q., Kliebenstein, D. J., Liu, S.-S., Fang, R.-X., and Ye, J. 2019. Viruses mobilize plant immunity to deter nonvector insect herbivores. Sci. Adv. 5:eaav9801. 